\title{
XPMaps and Topological Segmentation - A Unified Approach to Finite Topologies in the Plane
}

\author{
Ullrich Köthe \\ Cognitive Systems Group, University of Hamburg \\ koethe@informatik.uni-hamburg.de
}

\begin{abstract}
Finite topological spaces are now widely recognized as a valuable tool of image analysis. However, their practical application is complicated because there are so many different approaches. We show that there are close relationships between those approaches which motivate the introduction of $X P M a p s$ as a concept that subsumes the important characteristics of the other approaches. The notion of topological segmentations then extends this concept to a particular class of labelings of XPMaps. We show that the new notions lead to significant simplifications from both a theoretical and practical viewpoint.
\end{abstract}

\section{Introduction}

Many computer vision researchers now agree that the notion of finite topological spaces is very useful in image analysis and segmentation, as it allows for consistent descriptions of neighborhood relations, boundaries, and so on. Meanwhile, a number of different methods for the representation of finite topologies has been proposed, including cellular complexes [9], block complexes [10], the star topology [1], the Khalimsky grid [6, 3], combinatorial maps [15, 5] and border maps [2]. All methods approach the problem somewhat differently, but they have a lot in common as well. Unfortunately, the commonalties are not immediately apparent from the literature because most authors present their method in isolation or emphasize the differences to other methods. This makes it unnecessarily difficult to understand and compare the different approaches and to provide reusable implementations in a general image analysis framework.

In this contribution I'm going to propose a unified approach to finite topologies in the plane. I'll show that, under fairly general assumptions, different models can be transformed into each other. This will motivate the introduction of two generalized concepts (the XPMap and the topological segmentation) which subsume most aspects of the existing concepts. The concept of a XPMap is essentially a formalization of the border map introduced in [2].

This paper complements a previous paper 7] where solutions for some software design and implementation problems of the new approach were presented. Here, we look at the problem from a theoretical viewpoint in order to show formally why the underlying unification is possible and which properties it has. Due to space constraints, most proofs had to be skipped. They can be found in the long version [8] of this paper which is available from the author's WWW site. 

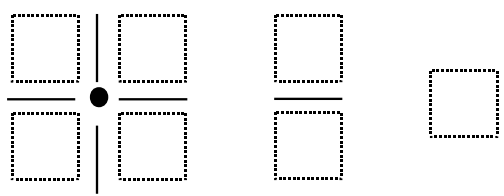

Fig. 1. The open stars of a vertex, arc and region (from left to right) in a square tessellation of the plane

\section{Models for Finite Topological Spaces in the Plane}

In this section, we will introduce the topological models we are going to analyze later. Here we only give definitions but do not yet discuss any relationships between them.

The most fundamental definition of a finite topological space is obtained on the basis of a complete division of the Euclidean plane. A plane division is a finite set of disjoint cells whose union completely covers the plane. There are three types of cells:

Vertices: $V=\left\{v_{1}, \ldots, v_{n}\right\}$ is a set of distinct points of the plane

Arcs: $A=\left\{a_{1}, \ldots, a_{m}\right\}$ is a set of disjoint open arcs whose end points are vertices from $V$. (An arc is a homeomorphic image of the interval $[0,1]$. An open arc is an arc less its end points. The two end points need not be distinct.)

Regions: $R=\left\{r_{1}, \ldots, r_{k}\right\}$ are the maximally connected components of the complement of the union of $V$ and $A$. Since $V \cup A$ is a closed set, all regions are open.

The Euclidean topology of the plane induces a neighborhood relation between the cells, which is used to define the smallest open neighborhood (the open star) of each cell. The set of open stars forms a basis for a finite topological space:

Definition 1: Let $T$ be a division of the Euclidean plane into cells (regions, arcs, vertices). Then the open star (smallest open neighborhood) of a cell $z$ is the set of cells that intersect with every Euclidean neighborhood of the points in $z$ :

$$
\operatorname{open}-\operatorname{star}(z \in T)=\left\{z^{\prime} \in T \mid \forall q \in z, \forall \varepsilon>0: B_{\varepsilon}(q) \cap z^{\prime} \neq \varnothing\right\}
$$

( $B_{\varepsilon}(q)$ denotes an open ball of radius $\varepsilon$ around the point $q$ ). The set of open stars is a basis of a finite topological space over the cells of the plane division.

Two divisions of the plane are topologically equivalent if there exists a 1-to-1 mapping between the cells of the division so that the open stars are preserved. Some authors, e.g. Ahronovitz et al. [1], further require all regions to be convex polytopes. We will not do this in this paper. The most common example for a star topology is obtained by tessellating the plane into equal squares. Then the open stars of the vertices, arcs, and regions look as depicted in figure 1 .

The biggest drawback of the plane division definition is its reliance on geometric concepts which prevents separation between topology and geometry. Therefore, a number of definitions not relying on the Euclidean space exist. The best known is the abstract cellular complex introduced to image analysis by Kovalevsky [9]:

Definition 2: A cell complex is a triple $(Z, \operatorname{dim}, B)$ where $Z$ is a set of cells, dim is a function that associates a non-negative integer dimension to each cell, and $B \subset Z \times Z$ is the bounding relation that describes which cells bound other cells. A cell may bound only cells of larger dimension, and the bounding relation must be transitive. If the largest dimension is $k$, we speak of a $k$-complex. 
A cell complex becomes a topological space by additionally defining the open sets as follows: a set of cells is called open if, whenever cell $z$ belongs to the set, all cells bounded by $z$ do also belong to the set. Kovalevsky proved that every finite topological space can be represented as a cell complex. In image analysis, we want to represent the topological structure of 2-dimensional images, so we are naturally interested in 2-complexes whose bounding relation is consistent with a division of the plane as defined above. We will call those planar cell complexes.

A special case of a cell complex is the block complex [10]. It is defined on a given cell complex by grouping cells into sets called block cells, or blocks for short. Blocks are defined by the property that they either contain a single 0-cell, or are homeomorphic to an open $k$-sphere. (A block is homeomorphic to an open k-sphere if it is isomorphic to a simply connected open set in some k-complex - see [10] for details.) A block's dimension equals the highest dimension of any of its cells.

Definition 3: A block complex is a complete partition of a given cell complex into blocks. The bounding relation of the blocks is induced by the bounding relation of the contained cells: a block bounds another one if and only if it contains a cell that bounds a cell in the other block.

Note that the induced bounding relation must meet the requirements of definition 2 . i.e. blocks may only bound blocks of smaller dimension, and the relation must be transitive. This means that all 1-blocks must be sequences of adjacent 0 - and 1-cells, and all junctions between 1-blocks must be 0-blocks.

Another approach to finite topological spaces originates from the field of graph theory - the combinatorial map [15, 5]:

Definition 4: A combinatorial map is a triple $(D, \sigma, \alpha)$ where $D$ is a set of darts (also known as half-edges), $\sigma$ is a permutation of the darts, and $\alpha$ is an involution of the darts.

In this context, a permutation is a mapping that associates to each dart a unique predecessor and a unique successor. By following successor chains, we can identify the cycles or orbits of the permutation. An involution is a permutation where each orbit contains exactly two elements. It can be shown that the mapping $\varphi=\sigma^{-1} \alpha$ is also a permutation. 11

The cycles of the $\sigma$ permutation are called the nodes of the map, the cycles of $\alpha$ are the edges, and the cycles of $\varphi$ the faces. A combinatorial map is planar (i.e. can be embedded in the plane) if its Euler characteristic equals two (cf. [15]):

$$
n-e+f=2
$$

(with $n, e$, and $f$ denoting the number of nodes, edges, and faces). Each planar combinatorial map has a unique dual map that is obtained by reversing the roles of the $\sigma$ and $\varphi$ orbits. It should be noted that the graph of a combinatorial map (i.e. the remains of the map when all faces are removed) must be connected, since each face is associated with exactly one orbit. Thus, faces in a combinatorial map may not have holes.

1 Combinatorial maps are sometimes defined by using four darts per edge ("quad-edges" or "crosses"), e.g. [15]. This allows the realization of non-orientable manifolds and the definition of generalized maps (G-maps) that can be generalized to arbitrary dimensions [12]. However, the half-edge definition suffices in the present context. 
The final approach we are going to deal with is Khalimsky's grid [6, 3]. It is defined as the product topology of two 1-dimensional topological spaces. In particular, one defines a connected ordered topological space (COTS) as a finite ordered set of points which alternate being open and closed. The smallest open neighborhood of an open point is the point itself, of a closed point it is the point and its predecessor and successor (in case of a closed endpoint, the neighborhood consists of two points only). Now Khalimsky's grid is defined like this:

Definition 5: A Khalimsky grid is defined as the space $X \times Y$ with the product topology, where $X$ and $Y$ are COTS with at least three points each.

The product topology defines the neighborhood of a grid point as $N((x, y))=N(x) \times N(y)$. When $x$ and $y$ are both open, the resulting point $(x, y)$ is also open. When they are closed, $(x, y)$ is closed as well, and its neighborhood consists of the 8 incident points. When an open point is combined with a closed point, a mixed point results, whose neighborhood contains the two incident closed points. These neighborhoods are analogous to the open stars in figure 1, with obvious modifications at the gird's border.

\section{Relationships between Finite Planar Topological Spaces}

In this section we show how the concepts defined above relate to each other by describing how a topological space represented in one concept can be translated into another. All missing proofs to the theorems can be found in [8].

Theorem 1: Any finite topology induced by a division of the plane can be represented by a planar cell complex.

The transformation is achieved by the following algorithm: Associate a 0-, 1- or 2-cell with every vertex, arc, and region respectively. Define the bounding relation so that cell $z$ bounds another cell $z$ ' whenever $z$ ' is part of the open star of $z$. In [8], we prove that the algorithm actually creates a valid cell complex.

The transformation in the opposite direction is not so simple because not every abstract cell complex corresponds to a division of the plane. We postpone this discussion until after the treatment of combinatorial maps because we will need a map as an intermediate representation during this transformation.

The transformation of a division of the plane into a combinatorial map is easy as long as the union of the vertices and arcs (the boundary set of the division) is a connected set. Otherwise, at least one region's boundary consists of several connected components, which is inconsistent with the requirement that every orbit of the $\varphi$ permutation corresponds to a distinct face. We will first discuss the transformation in the restricted case, and then extend the map definition for the general case.

Theorem 2: A finite topology induced by a division of the plane can be represented by a planar combinatorial map if the union of the arcs and vertices is connected.

To create a map from a division of the plane, first associate two darts with each arc, one for either direction. If the arc is defined by the function $a(t), 0<t<1$, one dart corresponds to $a(t)$ and the other to $a(1-t)$. The vertices at $a(0)$ and $a(1)$ are the start points of the darts respectively. Each pair of darts belonging to the same arc defines 
an orbit in the $\alpha$ involution. Then, for each vertex take the darts who start at that vertex, and sort them in the order induced by circling the vertex in mathematically positive direction. This gives the orbits of the $\sigma$ permutation. Under this definition the orbits of $\varphi=\sigma^{-1} \alpha$ indeed correspond to the regions of the plane division (see [8]). Two degenerate cases (not covered by the algorithm) require explicit definitions:

- the trivial map: it contains a single empty orbit in the $\varphi$ permutation and corresponds to the trivial division of the plane that contains only a single region.

- the vertex map: it contains a single empty orbit in both the $\sigma$ and $\varphi$ permutations and corresponds to one region that contains an isolated vertex.

In order to handle arbitrary divisions of the plane, including holes in regions, we have to extend the notion of planar combinatorial map. In particular, we must allow the graph of the extended map to be non-connected. Such a map is defined as follows:

Definition 6: An extended planar map (XPMap) is a tuple $\left(C, c_{0}\right.$, exterior, contains) where $C$ is a set of non-trivial planar combinatorial maps (the components of the XPMap), $c_{0}$ is a trivial map that represents the infinite face of the XPMap, exterior is a relation that labels one face of each component in $C$ as the exterior face, and contains is a relation that assigns each exterior face to exactly one non-exterior face or the infinite face.

Thus, if exterior face $f_{1}$ is contained in face $f_{2}$, the two faces are actually identical, and the component of $f_{1}$ becomes a hole in $f_{2}$. Euler's equation must be slightly modified for a XPMap with $k$ non-trivial components (that is, $k=|C|$ ):

$$
n-e+f-k=1
$$

Proof: This is easy to prove by induction. The trivial map has $f=1$ and $n=e=k=0$, the vertex map has $n=f=k=1, e=0$, and a connected map has $k=1$ and $n-e+f=2$, so the formula is valid for these cases. Suppose we have proved the formula for some $k$, i.e. $n^{(k)}-e^{(k)}+f^{(k)}-k=1$. Now add a new component with $n^{(1)}-e^{(1)}+f^{(1)}-1=1$. We may add the two equations together: $n^{(k)}+n^{(1)}-e^{(k)}-e^{(1)}+f^{(k)}+f^{(1)}-k-1=2$. It holds that $n^{(k)}+n^{(1)}=n^{(k+1)}$, $e^{(k)}+e^{(1)}=e^{(k+1)}$, but $f^{(k)}+f^{(1)}=f^{(k+1)}+1$, because the exterior face of the newly added component has already been present in the existing XPMap and must not be counted twice. Thus, $n^{(k+1)}-e^{(k+1)}+f^{(k+1)}-(k+1)=1$ as claimed.

It should be noted that XPMaps are essentially equivalent to the border maps proposed by Bertrand et al. [2] (but their paper lacks a formal definition). Their approach is based on G-maps because they are interested in 2- and 3-dimensional problems. Since this is not needed here, our definition is sufficient.

It is now possible to construct an XPMap from an arbitrary division of the plane: First take each connected component of the division's boundary set and create the corresponding combinatorial map. These maps become the components of the XPMap. Each component has exactly one $\varphi$ orbit that is traversed in mathematically negative orientation which becomes the exterior face. Now construct the containment relation according to the inclusion of components within regions of the plane division.

To go in the opposite direction, we must find a plane imbedding of a given XPMap. This problem is part of the standard problem of drawing a planar graph, suitable algorithms can be found in [4].

It is now easy to see that any XPMap can be transformed into a cell complex:

Theorem 3: Any XPMap defines a corresponding cell complex. 
Proof: For every node, edge, and face of the map create a corresponding 0-, 1-, or 2-cell, respectively. Let a 1-cell bound a 2-cell whenever a dart of the corresponding edge is part of any $\varphi$ orbit associated with the corresponding face. Let a 0 -cell bound a 1-cell whenever a dart of the edge is part of the corresponding node's $\sigma$ orbit. Let a 0 -cell bound a 2-cell whenever its $\sigma$ orbit contains a dart whose corresponding 1-cell bounds that 2-cell. The bounding relation obviously conforms to definition 2 .

The bounding relation of the cells induces an equivalent bounding relation of the nodes, edges, and faces. By extension of terminology, and we will say that a node (edge) bounds an edge (face) if the corresponding 0-cell (1-cell) bounds the corresponding 1-cell (2-cell). The bounding relation defines the topology of an XPMap:

Definition 7: A set of nodes, edges, and faces in a XPMap is called open if the corresponding cells form an open set in the corresponding cell complex. The open star of a node, edge, or face (the smallest open set containing this entity) is the set containing the entity itself and all entities bound by it.

Transformations of cell complexes into XPMaps are more difficult. A 2-complex is not necessarily embeddable into the plane - it might correspond to a non orientable surface or a surface of higher genus. So, some necessary conditions must be met. First, one 2-cell must be denoted the infinite face (it can be chosen arbitrarily). Second, Euler's formula 2 must be fulfilled (again, $k$ is the number of components of the cell complex' graph, i.e. the remains after removing all 2-cells). Third, all 1 -cells must be bounded by one or two 0 -cells since all darts must be incident to a node. Unfortunately, its not clear to me whether these requirements are sufficient.

Even when these requirements are fulfilled, the transformation of a 2-complex to a XPMap is not necessarily unique. If the cell complex' graph is 1-connected ${ }^{2}$ or disconnected, the neighborhood relation does not change when certain sub-graphs are replaced by their mirror images (see figure 2 for examples). In these cases, the order of the darts within each $\sigma$ or $\varphi$ orbit is not uniquely determined.

An algorithm that produces one possible XPMap out of a given planar cell complex is given in [8]. This algorithm terminates with no free darts remaining, if the cell complex is planar. The algorithm implies a method for generating a planar imbedding of a cell complex: first transform the cell complex into an XPMap, and then imbed the map into the plane by a standard imbedding algorithm (see [4]). However, although the algorithm may be interesting from a theoretical point of view, its practical value is probably limited: in practice, a cell complex will usually be equipped with additional geometric information that uniquely determines the correct order of the darts in the orbits and the imbedding into the plane.

To allow the establishment of relationships between Khalimsky's grid and the other topological spaces, we must modify the definition of the grid somewhat so that it gets an infinite point that can be mapped to the infinite face of the map:

Definition 8: A Khalimsky grid with infinite point is defined as follows: the COTS $X$ and $Y$ constituting the grid must be chosen so that their first and last points are all closed. Then the border of the grid consists only of closed and mixed points. Add an additional point - the infinite point - that by definition belongs to the neighborhood of all border points.

\footnotetext{
${ }^{2} \mathrm{~A}$ graph is $k$-connected if at least $k$ vertices must be removed to make the graph disconnected.
} 

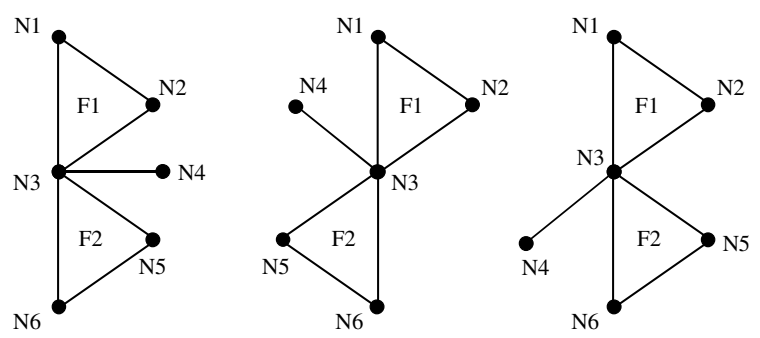

Fig. 2. Three imbeddings of the same cell complex that differ in the ordering of the darts in the $\sigma$ and $\varphi$ orbits. Note that the cell complex' graph is only 1-connected.

This extension allows formulation of the following theorem:

Theorem 4: A Khalimsky grid with infinite point defines a corresponding XPMap.

The transformation is obtained as follows: First, we associate a node with every closed point, an edge with every mixed one, and a face with every open one. Then we create two darts for each mixed point, starting at either incident closed point. Each pair forms an orbit of the $\alpha$ involution. It is convenient to identify each dart by the topological coordinates of its start point and the relative orientation between the start and mixed point (east, north, west, and south). A $\sigma$ permutation is now defined by taking the darts associated with the same start point and sorting them according to their orientation. This choice also implies the expected $\varphi$ orbits, as is shown in [8].

In contrast, a combinatorial map can only be represented as a Khalimsky grid if it has the required regular structure: all nodes and faces must be adjacent to exactly four darts (with obvious modifications at the exterior border). Since this is a rather strong limitation, Khalimsky's grid itself is not useful for the representation of arbitrarily shaped topological spaces. Instead, one builds block complexes on top of the grid. That is, one creates irregularly shaped cells by grouping the grid points into appropriate blocks. We will discuss this in detail in section 5 .

\section{Euler Operators}

Before we can turn our attention to block complexes, we must define and discuss Euler operators 13. Euler operators turn a given planar topological structure into a different one. The term stems from the restriction that Euler's equation must remain valid in the new structure, so that planarity is preserved (some authors call such operators superficial). The proposed Euler operators are elementary in the sense that they change the number of nodes, edges, faces, and components by at most one. They are complete in the sense that any desired transformation can be expressed as a sequence of Euler operations. Completeness follows from theorem 5 below: every XPMap is an open set and can be reduced to a single face by a sequence of Euler operations. By reversing the sequence, any XPMap can be built out of the trivial map.

The operators will be described in the context of XPMaps only. The proofs which check that Euler's equation indeed remains valid are again found in [8]. Analogous operators in other topological models are implied by the transformations already discussed. For example, figure 3 shows how the operators to be defined act on a division of the plane. Of course, application of Euler operators to the Khalimsky grid 

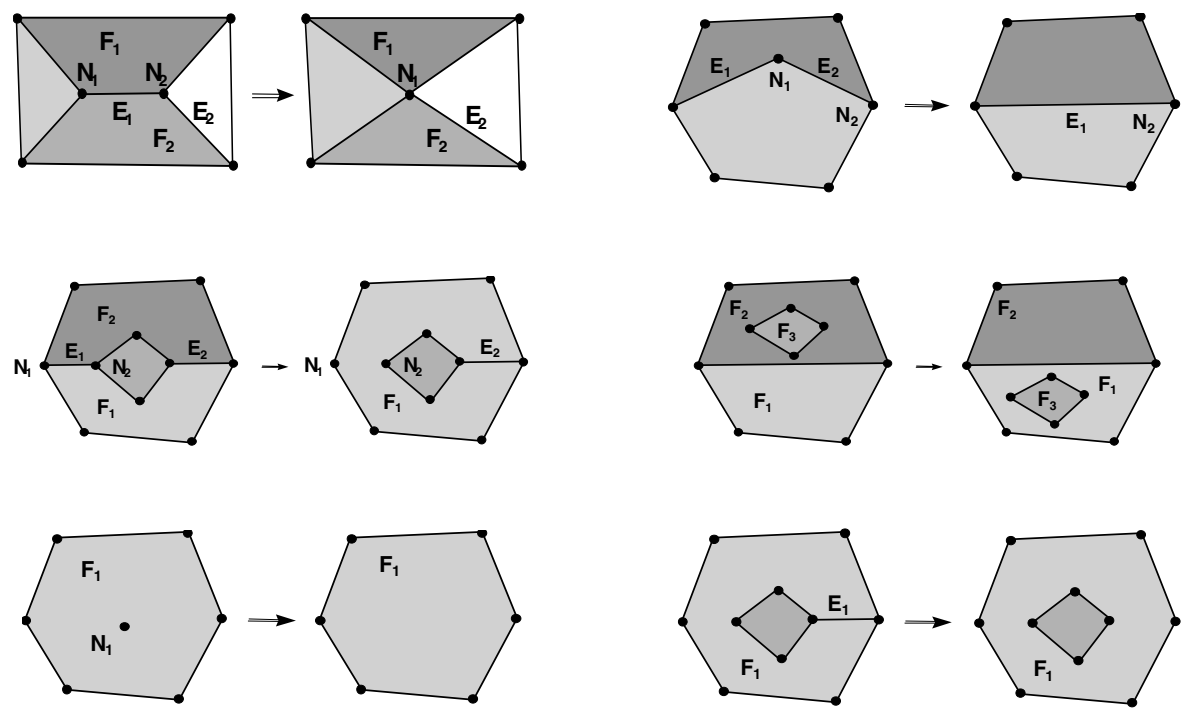

Fig. 3. Examples for Euler operators. first row: edge contraction and merge edges, second row: merge faces and move component, third row: remove isolated node and remove bridge

is forbidden because the grid's structure is fixed. We will consider two kinds of operators: first, those that do not change the number of components of the map's graph (so that we can restrict the discussion to standard combinatorial maps), and second the ones that do (and thus require a XPMap).

Node Split and Edge Contraction. A node split creates two nodes from one. Some existing edges are moved to the new node, and a new edge connecting the nodes is added. The split must be arranged so that the order of the edges in the orbits is not changed (formal definition below). The inverse operation is called edge contraction, because the two ends of an edge are contracted into a single node.

Definition 9 (node split): Suppose the node to be split is associated with the orbit $\sigma_{1}=\left(d_{0}, \ldots, d_{n-1}\right)$. Remove $\sigma_{1}$ from the set of orbits and chose darts $d_{i}$ and $d_{j}$ $(0 \leq i \leq j<n)$. Split the orbit after these darts into the sequences $\left(d_{j+1}, \ldots, d_{i}\right)$ and $\left(d_{i+1}, \ldots, d_{j}\right)$ where addition is taken modulo $n$. If $i=j$, the second sequence is empty. Create a new pair of darts $d, d^{\prime}$ and insert the pair as a new orbit into $\alpha$. Add one new dart to either sequence and complete them to the new orbits $\sigma_{1}{ }^{\prime}=\left(d_{j+1}, \ldots, d_{i}, d\right)$ and $\sigma_{1}{ }^{\prime}=\left(d_{i+1}, \ldots, d_{j}, d^{\prime}\right)$ which are inserted into the set of orbits. For the inverse operation edge contraction, the steps are reversed. The edge to be contracted must not be a self-loop.

By definition, the operator increases the number of orbits in the $\sigma$ and $\alpha$ permutation by one. The number of components and $\varphi$ orbits does not change since the newly inserted edge ensures that the graph of the map remains connected (proof in [8]). 
Edge Split and Edge Merge. Edge split creates two edges by placing a new node into an existing edge. Edge merge reverses this operation. Merging may only be applied to a pair of distinct edges whose common node has degree 2 (otherwise, forbidden configurations would arise). Edge split is a special case of node split (so we omit the formal definition here). Nonetheless, it is useful to define edge split as an operator of its own because of its special semantic.

Face Merge and Face Split. The face merge operator removes an edge, so that its adjacent faces are merged into one. Face split is the inverse operation. The edge to be removed must bound two distinct faces (i.e. it must not be a bridge) as otherwise the number of components would be increased (bridges are discussed below).

Definition 10 (face split): Create a new pair of darts $d$ and $d$ ' and insert the pair as a new orbit into $\alpha$. Select two darts $d_{1}$ and $d_{2}$ which belong to the same $\varphi$ orbit. Insert $d$ after $d_{1}$ and $d^{\prime}$ after $d_{2}$ into the respective $\sigma$ orbits. Face merge is executed be reversing those steps, provided that the edge $\left(d, d^{\prime}\right)$ is not a bridge.

Face split is the dual operation of node split: it is equivalent to node split in the dual map.

Move Component. This operation is very simple and does not involve any orbits:

Definition 11 (move component): Take an entry of an XPMap's containment relation and replace the enclosing face by another one (which must not itself be an exterior face).

This operation is especially useful in the context of splitting faces with holes: if the face to be split contains some components of the XPMap, the definition of the face split operator doesn't tell which of the newly created faces will contain those components afterwards. Suitable adjustments can be made by means of "move component".

Insert/Remove Isolated Node. This operation is the simplest possibility to create a new component within an XPMap:

Definition 12 (insert isolated node): Create a new vertex map. Make its single face the exterior one and associate it with an arbitrary non-exterior face by adding appropriate entries to the XPMap's exterior and contains relations.

Remove/Insert Bridge. A bridge is an edge that bounds only one face. Removing a bridge from the $\alpha$ and $\sigma$ orbits is simple, but modification of the $\varphi$ orbits is rather involved. This stems form the fact that "remove bridge" creates a new component, and it is not trivial to decide which $\varphi$ orbit becomes the exterior face of the new component. Due to space constraints, the algorithm must be skipped, see again [8].

\section{Block Complexes and Topological Segmentation}

In definition 3, block complexes are defined on top of cell complexes. Although we could extend this definition to other topological structures, we will not do so because the restriction " $k$-blocks must be homeomorphic to open $k$-spheres" is not acceptable in the context of image analysis: it requires all regions to be simply connected (i.e. holes in regions are not allowed) and each 0-block to consist of a single 0-cell (and thus the maximum degree of 0-blocks is bounded by the 0-cell's degree - so junctions 

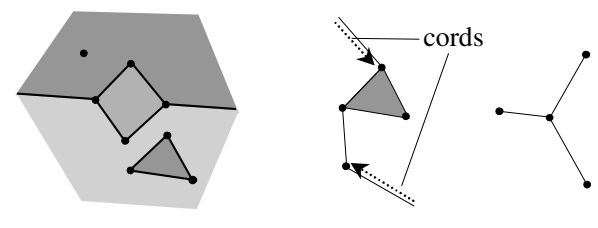

Fig. 4. Left: examples for 2-, 1-, and 0-segments (left to right), right: topological segmentation of a Khalimsky grid with infinite point

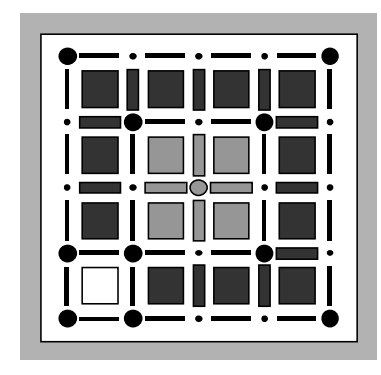

of degree $>4$ cannot be represented on top of a Khalimsky grid). Therefore, we will develop a more general concept for grouping the cells of a digital topological structure which we will call a topological segmentation. A topological segmentation is a complete partition of a digital topological space into $k$-segments $(k=0,1,2)$ such that every $k$-segment can be transformed into a single $k$-cell by a suitable sequence of Euler operations. Before we can give a formal definition we need some preliminaries:

Theorem 5: Any connected open set in a XPMap can be reduced to a single face by a sequence of Euler operators, without changing the structure of the set's complement. Any simply connected closed set can be reduced to a single node.

The proof can be found in [8]. Furthermore, we need the notion of hammocks:

Definition 13: A hammock is the union of a simply connected closed set and two edges emanating from the set such that removal of these edges would turn the closed set into a new component of the XPMap. An edge is said to emanate from a set if one of its end nodes belongs to the set but not the other. The darts starting at these external nodes are called the cords of the hammock.

Hammocks are useful because they can be reduced to single edges by a sequence of Euler operations: reduce the closed set in the hammock to a single node which is bound by the two remaining edges of the hammock, but no other edge. Then apply the operator "merge edges" to turn the remaining cells into a single edge.

Definition 14: A topological segmentation is a complete partition of an underlying XPMap into $k$-segments $(k=0,1,2)$ such that every 0 -segment is a simply connected closed set, every 1 -segment is a hammock, and every 2 -segment is a connected open set.

Figure 4 shows examples of $k$-segments and a topological segmentation. According to the discussion above, any 0 -segment can be reduced to a single node, any 1-segment to a single edge, and any 2-segment to a single face. By reducing all segments we arrive at a new XPMap which we will call the reduced XPMap of the topological segmentation. Definition 14 replaces the restriction "every $k$-block must be homeomorphic to an open $k$-cell" by "every $k$-segment must be reducible to a single $k$-cell". Therefore, a topological segmentation may contain 2-segments with holes and 0 -segments that have higher degree than any node of the underlying XPMap.

Although, in principle every topological segmentation can be reduced to a XPMap, it is not necessary to actually perform the reduction. Instead, we can just label the segments in the underlying XPMap and work with the segments directly. In fact, this 
is the only possibility to represent irregular divisions of the plane by means of a Khalimsky grid: since the grid's structure is rigid, one cannot perform Euler operations on it, but one can still label points into segments.

In order to make a labeled XPMap or Khalimsky grid behave like a reduced XPMap, we must specify the orbits of the topological segmentation. To avoid confusion, we will denote these orbits by Greek capitals $A, \Phi, \Sigma$, in contrast to the lower case $\alpha, \varphi$, and $\sigma$ orbits of the underlying XPMap. The cords of the hammocks are the basis of the orbit definitions. These definitions amount to contour following algorithms that go around segments in order to find all incident cords. Efficient and elegant implementations of these algorithms can be obtained by means of generic programming, as described in [7]:

$A$ orbits of a topological segmentation: The $A$ orbits associate the two cords of a hammock: given one cord, we want to find the other. To do so, observe that the left face of the first cord is the right face of the second. Thus, we can follow the $\varphi$ orbit of the first cord until we arrive at a dart that doesn't belong to the present hammock. Its $\sigma$-successor is the sought for $A$ successor of the first cord.

$\Sigma$ orbits of a topological segmentation: $\Sigma$ orbits associate cords with 0-segments. This is easy if the 0 -segment consists of a single node: then the $\Sigma$ orbit is obtained from the node's $\sigma$ orbit by simply dropping the darts that are not cords. In case of a more complicated 0-segment, the principle is the same, but instead of just considering a single $\sigma$ orbit we must now follow the entire contour of the segment. The following algorithm finds the $\Sigma$ successor of a given cord:

1. Find the $\sigma$ successor of the present dart. If the successor is a cord, stop. If it belongs to an edge that is part of the 0 -segment, go to 2 . Otherwise go to 1 .

2 . Find the $\alpha$ successor of the present dart, and find the $\sigma$ successor of the resulting dart. If this dart is a cord, stop. If it belongs to an edge that is part of the 0 -segment, go to 2 . Otherwise go to 1 .

$\Phi$ orbits of a topological segmentation: The $\Phi$ orbits associate cords with 2-segments. The $\Phi$ successor of a given cord is found as follows: go to the $A$ successor of the present cord, and find its $\Sigma$ predecessor. Thus, $\Phi=\Sigma^{l} A$.

It can be shown that the orbits of a topological segmentation are equivalent to the corresponding orbits in the reduced XPMap. Furthermore, we can define Euler operators for a topological segmentation. They perform the same logical operation as their XPMap equivalent, but do not merge cells. Instead, they re-label the segments in order to reflect the modified structure. The only difference to XPMaps is that split operations can only be applied as long as the segments contain more than one cell.

\section{Conclusions}

In this paper, we have presented a novel, unified approach to the representation of finite topologies in the plane. XPMaps incorporate the full range of functionality from cell complexes, combinatorial maps and plane divisions. The concept of a topological segmentation allows to define labelings of XPMaps that again have the properties of a 
topological space. This provides a smooth transition from the pixel plane to an abstract topological space as it allows for the definition of irregular topologies on top of regular grids. Furthermore, by applying labeling and reduction repeatedly, a wide variety of irregular pyramids can be realized. This idea leads to a generalization of concepts form [11, 14] and will be explored in an upcoming paper.

Of course, it would be desirable to carry the analysis to 3-dimensional spaces. However, given the wealth of unsolved problems in 2D image analysis and segmentation, its appears equally worthwhile to utilize the power of the new approach here. The generalized formal definitions presented provide a firm basis for studying the relationships between topology and the imaging process. By using XPMaps as a common representation for segmentation results, we will be able to develop more robust segmentation algorithms and achieve a unification of approaches in general.

\section{References}

[1] E. Ahronovitz, J.P. Aubert, C. Fiorio: "The Star Topology: a Topology for Image Analsysis”, Proc. $5^{\text {th }}$ Intl. Conf. Discrete Geometry for Computer Imagery, DGCI'95, 1995

[2] Y. Bertrand, C. Fiorio, Y. Pennaneach: "Border Map: a Topological Representation for nD Image Analysis”, G. Bertrand, M. Couprie, L. Perroton (eds.): Proc. $8{ }^{\text {th }}$ Intl. Conf. Discrete Geometry for Computer Imagery, DGCI'99, Springer LNCS 1568, 1999

[3] J.-P. Braquelaire, J.-P. Domenger: "Representation of Region Segmented Images with Discrete Maps", Université Bordeaux, Laboratoire Bordelais de Recherche en Informatique, Technical Report 1127-96, 1996

[4] G. Di Battista, P. Eades, R. Tamassia, I.G. Tollis: "Graph Drawing”, Prentice Hall, 1999

[5] J.-F. Dufourd, F. Puitg: "Functional specification and prototyping with oriented combinatorial maps", Computational Geometry 16 (2000) 129-156

[6] E. Khalimsky, R. Kopperman, P. Meyer: "Computer Graphics and Connected Topologies on Finite Ordered Sets", J. Topology and its Applications, vol. 36, pp. 1-27, 1990

[7] U. Köthe: "Generic Programming Techniques that Make Planar Cell Complexes Easy to Use”, G.Bertrand, A.Imiya, R.Klette (eds.): Proc. of Dagstuhl WS on Digital and Image Geometry 2000, Springer LNCS 2243, 2001

[8] U. Köthe: "XPMaps and Topological Segmentation - a Unified Approach to Finite Topologies in the Plane (long version)", Uni Hamburg, Preprint FBI-HH-M-308/0, 2001 http://kogs-www.informatik.uni-hamburg.de/ koethe/papers/XPMapsLong.ps.gz

[9] V. Kovalevsky: "Finite Topology as Applied to Image Analysis", Computer Vision, Graphics, and Image Processing, 46(2), pp. 141-161, 1989

[10] V. Kovalevsky: "Computergestützte Untersuchung topologischer Eigenschaften mehrdimensionaler Räume”, Preprints CS-03-00, Computer Science Department, University of Rostock, 2000 (in German)

[11] W. Kropatsch: "Building Irregular Pyramids by Dual Graph Contraction”, IEE Proceedings - Vision, Image and Signal Processing, 142(6), 366-374, 1995

[12] P. Lienhardt: "Topological models for boundary representation: a comparison with ndimensional generalized maps", computer aided design, 23(1), 59-82, 1991

[13] M. Mäntylä: “An Introduction to Solid Modeling”, Computer Science Press, 1988

[14] A. Montanvert, P. Meer, A. Rosenfeld: "Hierarchical Image Analysis Using Irregular Tessellations”, IEEE Trans. Pattern Anal. and Machine Intelligence, 13(4), 307-316, 1991

[15] W.T. Tutte: “Graph Theory”, Cambridge University Press, 1984 